\title{
Transition between Instability and Seeded Self-Modulation of a Relativistic Particle Bunch in Plasma
}

F. Batsch $\odot,{ }^{1}$ P. Muggli, ${ }^{1}$ R. Agnello, ${ }^{2}$ C. C. Ahdida, ${ }^{3}$ M. C. Amoedo Goncalves, ${ }^{3}$ Y. Andrebe,${ }^{2}$ O. Apsimon, ${ }^{4,5}$ R. Apsimon, ${ }^{4,6}$ A.-M. Bachmann, ${ }^{1}$ M. A. Baistrukov, ${ }^{7,8}$ P. Blanchard, ${ }^{2}$ F. Braunmüller, ${ }^{1}$ P. N. Burrows, ${ }^{9}$ B. Buttenschön, ${ }^{10}$ A. Caldwell, ${ }^{1}$ J. Chappell, ${ }^{11}$ E. Chevallay, ${ }^{3}$ M. Chung, ${ }^{12}$ D. A. Cooke, ${ }^{11}$ H. Damerau, ${ }^{3}$ C. Davut, ${ }^{4,13}$ G. Demeter, ${ }^{14}$ H. L. Deubner, ${ }^{15}$ S. Doebert, ${ }^{3}$ J. Farmer, ${ }^{1,3}$ A. Fasoli, ${ }^{2}$ V. N. Fedosseev, ${ }^{3}$ R. Fiorito, ${ }^{4,5}$ R. A. Fonseca, ${ }^{16,17}$ F. Friebel, ${ }^{3}$ I. Furno, ${ }^{2}$ L. Garolfi, ${ }^{18}$ S. Gessner, ${ }^{3,19}$ I. Gorgisyan, ${ }^{3}$ A. A. Gorn, ${ }^{7,8}$ E. Granados, ${ }^{3}$ M. Granetzny, ${ }^{20}$ T. Graubner, ${ }^{15}$ O. Grulke, ${ }^{10,21}$ E. Gschwendtner, ${ }^{3}$ V. Hafych, ${ }^{1}$ A. Helm, ${ }^{17}$ J. R. Henderson, ${ }^{4,22}$ M. Hüther, ${ }^{1}$ I. Yu. Kargapolov, ${ }^{7,8}$ S.-Y. Kim, ${ }^{12}$ F. Kraus, ${ }^{15}$ M. Krupa, ${ }^{3}$ T. Lefevre, ${ }^{3}$ L. Liang, ${ }^{4,13}$ S. Liu, ${ }^{18}$ N. Lopes,${ }^{17}$ K. V. Lotov, ${ }^{7,8}$ M. Martyanov, ${ }^{1}$ S. Mazzoni, ${ }^{3}$ D. Medina Godoy, ${ }^{3}$ V. A. Minakov, ${ }^{7,8}$ J. T. Moody, ${ }^{1}$ K. Moon, ${ }^{12}$ P. I. Morales Guzmán, ${ }^{1}$ M. Moreira, ${ }^{3,17}$ T. Nechaeva, ${ }^{1}$ E. Nowak, ${ }^{3}$ C. Pakuza, ${ }^{9}$ H. Panuganti, ${ }^{3}$ A. Pardons, ${ }^{3}$ A. Perera,${ }^{4,5}$ J. Pucek, ${ }^{1}$ A. Pukhov, ${ }^{23}$ R. L. Ramjiawan, ${ }^{3,9}$ S. Rey, ${ }^{3}$ K. Rieger, ${ }^{1}$ O. Schmitz, ${ }^{20}$ E. Senes,${ }^{3,9}$ L. O. Silva, ${ }^{17}$ R. Speroni, ${ }^{3}$ R. I. Spitsyn, ${ }^{7,8}$ C. Stollberg, ${ }^{2}$ A. Sublet, ${ }^{3}$ A. Topaloudis, ${ }^{3}$ N. Torrado, ${ }^{17}$ P. V. Tuev, ${ }^{7,8}$ M. Turner,,${ }^{3,24}$ F. Velotti, ${ }^{3}$ L. Verra, ${ }^{1,3,25}$ V. A. Verzilov, ${ }^{18}$ J. Vieira, ${ }^{17}$ H. Vincke, ${ }^{3}$ C. P. Welsch, ${ }^{4,5}$ M. Wendt, ${ }^{3}$ M. Wing, ${ }^{11}$ P. Wiwattananon, ${ }^{3}$ J. Wolfenden, ${ }^{4,5}$ B. Woolley, ${ }^{3}$ G. Xia, ${ }^{4,13}$ M. Zepp, ${ }^{20}$ and G. Zevi Della Porta ${ }^{3}$

(AWAKE Collaboration)

\author{
${ }^{1}$ Max Planck Institute for Physics, Munich, Germany \\ ${ }^{2}$ Ecole Polytechnique Federale de Lausanne (EPFL), Swiss Plasma Center (SPC), Lausanne, Switzerland \\ ${ }^{3}$ CERN, Geneva, Switzerland \\ ${ }^{4}$ Cockcroft Institute, Daresbury, United Kingdom \\ ${ }^{5}$ University of Liverpool, Liverpool, United Kingdom \\ ${ }^{6}$ Lancaster University, Lancaster, United Kingdom \\ ${ }^{7}$ Novosibirsk State University, Novosibirsk, Russia \\ ${ }^{8}$ Budker Institute of Nuclear Physics SB RAS, Novosibirsk, Russia \\ ${ }^{9}$ John Adams Institute, Oxford University, Oxford, United Kingdom \\ ${ }^{10}$ Max Planck Institute for Plasma Physics, Greifswald, Germany \\ ${ }^{11}$ University College London, London, United Kingdom \\ ${ }^{12}$ Ulsan National Institute of Science and Technology, Ulsan, Republic of Korea \\ ${ }^{13}$ University of Manchester, Manchester, United Kingdom \\ ${ }^{14}$ Wigner Research Center for Physics, Budapest, Hungary \\ ${ }^{15}$ Philipps-Universität Marburg, Marburg, Germany \\ ${ }^{16}$ ISCTE-Instituto Universitéario de Lisboa, Portugal \\ ${ }^{17}$ GoLP/Instituto de Plasmas e Fusão Nuclear, Instituto Superior Técnico, Universidade de Lisboa, Lisbon, Portugal \\ ${ }^{18}$ TRIUMF, Vancouver, Canada \\ ${ }^{19}$ SLAC National Accelerator Laboratory, Menlo Park, California, USA \\ ${ }^{20}$ University of Wisconsin, Madison, Wisconsin, USA \\ ${ }^{21}$ Technical University of Denmark, Lyngby, Denmark \\ ${ }^{22}$ Accelerator Science and Technology Centre, ASTeC, STFC Daresbury Laboratory, Warrington, United Kingdom \\ ${ }^{23}$ Heinrich-Heine-Universität Düsseldorf, Düsseldorf, Germany \\ ${ }^{24}$ Lawrence Berkeley National Laboratory, Berkeley, California, USA \\ ${ }^{25}$ Technical University Munich, Munich, Germany
}

(Received 18 December 2020; revised 18 February 2021; accepted 9 March 2021; published 20 April 2021)

We use a relativistic ionization front to provide various initial transverse wakefield amplitudes for the self-modulation of a long proton bunch in plasma. We show experimentally that, with sufficient initial amplitude $[\geq(4.1 \pm 0.4) \mathrm{MV} / \mathrm{m}]$, the phase of the modulation along the bunch is reproducible from event to event, with $3 \%-7 \%$ (of $2 \pi$ ) rms variations all along the bunch. The phase is not reproducible for lower

Published by the American Physical Society under the terms of the Creative Commons Attribution 4.0 International license. Further distribution of this work must maintain attribution to the author(s) and the published article's title, journal citation, and DOI. 
initial amplitudes. We observe the transition between these two regimes. Phase reproducibility is essential for deterministic external injection of particles to be accelerated.

DOI: 10.1103/PhysRevLett.126.164802

Introduction.-Accelerators rely on precise control of parameters to produce high-quality, high-energy particle bunches for numerous applications. A class of novel accelerators using plasma as a medium to sustain large accelerating [1,2] and focusing [3] fields has emerged and has made remarkable experimental progress over the past two decades [4-6].

Most of these accelerators use a very short $(<1 \mathrm{ps})$, intense laser pulse [1] or a dense, relativistic particle bunch [2] to drive wakefields in plasma. The amplitude of the accelerating field that can be sustained with a plasma of electron density $n_{e 0}$ is on the order of the wave breaking field [7]: $E_{\mathrm{WB}}=\left(m_{e} c / e\right) \omega_{p e}$. Here $\omega_{p e}=\left(n_{e 0} e^{2} / \varepsilon_{0} m_{e}\right)^{1 / 2}$ is the plasma electron angular frequency [8]. Assuming the driver of rms duration $\sigma_{t}$ fits within the structure, i.e., $\sigma_{t} \cong 1 / \omega_{p e}$, one can rewrite: $E_{\mathrm{WB}}=\left(m_{e} c / e\right)\left(1 / \sigma_{t}\right)$. Therefore, operating at high accelerating field $(>1 \mathrm{GV} / \mathrm{m})$ requires high plasma density and short $(<2 \mathrm{ps})$ pulses or bunches with similarly small radii $\left(\sigma_{r 0} \leq c / \omega_{p e} \leq 600 \mu \mathrm{m}\right)[9]$.

The system extracts energy from the driver and transfers it to a witness bunch, through the plasma. The total energy gain of the witness bunch is limited to the energy carried by the driver. Short laser pulses and particle bunches available today and suitable to drive $>1 \mathrm{GV} / \mathrm{m}$ amplitude wakefields carry less than $\sim 100 \mathrm{~J}$ of energy. Laser pulses and particle bunches carrying much more energy are too long, typically $>100 \mathrm{ps}$, to drive large amplitude wakefields when following the above $E_{\mathrm{WB}} \propto 1 / \sigma_{t}$ scaling. However, long laser pulses [10] and long, relativistic particle bunches [11] propagating in dense plasma, i.e., $\sigma_{t} \gg 1 / \omega_{p e}$, are subject to self-modulation (SM) instabilities. These instabilities can transform them into a train of pulses or bunches shorter than, and with a periodicity of $2 \pi / \omega_{p e}$. The train can then resonantly excite large amplitude wakefields. Control of the SM process, in particular of the relative phase of the wakefields, is necessary to deterministically inject a witness bunch shorter than $1 / \omega_{p e}$ into the accelerating and focusing phase of the wakefields.

As the first proton-driven plasma wakefield acceleration experiment, AWAKE $[12,13]$ recently demonstrated that the SM process does indeed transform a long proton bunch $\left(\sigma_{t}>200 \mathrm{ps}\right)$ into a train of microbunches with period $2 \pi / \omega_{p e}(<10 \mathrm{ps})$ [14]. We also demonstrated that the process grows along the bunch and along the plasma, from the initial wakefield amplitude, to saturate at much larger values $[15,16]$. Electrons were externally injected in the wakefields, though without phase control (electron bunch duration on the order of $2 \pi / \omega_{p e}$ ) and accelerated from $\sim 19 \mathrm{MeV}$ to $\sim 2 \mathrm{GeV}$ [17]. For this scheme to become an accelerator that can produce not only sufficiently highenergy particles, but also sufficiently high-quality bunches in terms of high population, low energy spread, and low emittance [18], one needs to show that the SM process can be controlled.

Seeding of SM in the sense of triggering the start of its growth has been demonstrated experimentally with a relativistic ionization front (RIF) in a long pulse, laserdriven plasma wakefield accelerator [19], and with the sharp density front of a long electron bunch in a particledriven plasma wakefiled accelerator [20]. However, measurements on the effect of that seeding on the phase of growing wakefields have not been reported. As demonstrated below, triggering the SM is not sufficient to ensure the reproducibility of the phase of the wakefields from event to event.

In this Letter, we demonstrate experimentally for the first time that the phase of the SM of a long, relativistic particle bunch can be controlled by seeding the process with a RIF. This means that we define seeding as the conditions leading to a reproducible timing or phase of the SM along the bunch with respect to the RIF from event to event. From time-resolved images of the bunch obtained at two plasma densities, we analyze the relative timing or phase of the microbunches along the proton bunch, after the plasma. We control the initial wakefield amplitude through the timing of the RIF along the bunch. When the process is not seeded, we observe randomly distributed phases and thus the SM instability (SMI) [11]. With sufficiently strong initial amplitude, the phase of the wakefields varies by only a small fraction of $2 \pi$ from event to event, the characteristic of seeded SM (SSM) [13]. This is despite natural variations of the incoming bunch parameters [21]. We thus observe the transition from SMI to SSM. We also observe phase reproducibility over more than $2 \sigma_{t}$ along the bunch. Phase reproducibility is essential for future experiments [13] with deterministic, external injection of particles to be accelerated $\left(e^{-}\right.$or $\left.e^{+}\right)$at a precise phase within the accelerating and focusing region of the wakefields [18].

Experimental results presented here show that the phase of the self-modulation instability, a fundamental beamplasma interaction mechanism [11], can be controlled. It is also a requirement for future acceleration experiments.

Experimental setup.-The CERN Super Proton Synchrotron (SPS) provides a Gaussian bunch with $400 \mathrm{GeV}$ energy per proton, $3 \times 10^{11}$ particles, and a rms duration $\sigma_{t}=250 \mathrm{ps}$. The bunch enters a 10-m-long vapor source [22,23], as shown in Fig. 1, with rms waist size $\sigma_{r 0}=150 \mu \mathrm{m}$. The source contains rubidium $(\mathrm{Rb})$ vapor 
with density $n_{\mathrm{Rb}}$ adjustable in the $(0.5-10) \times 10^{14} \mathrm{~cm}^{-3}$ range and with uniform temperature and thus density distributions $\left(\Delta n_{\mathrm{Rb}} / n_{\mathrm{Rb}}=\Delta T / T<0.2 \%\right.$ [23]). The vapor density is measured to better than $0.5 \%$ [24] at both ends of the source. A Ti:sapphire laser system provides a 120 fs, $\leq 450 \mathrm{~mJ}$ laser pulse that can serve two purposes. First, when propagating along the vapor column it creates the plasma at the RIF. The RIF transforms the Rb vapor into a $\sim 2 \mathrm{~mm}$ diameter plasma with density and uniformity equal to those of the vapor [14]. Therefore, hereafter we quote the corresponding plasma density instead of the measured $\mathrm{Rb}$ vapor density $\left(n_{e 0}=n_{\mathrm{Rb}}\right)$. Second, when propagating within the proton bunch, the RIF triggers the sudden $\left(\ll 1 / \omega_{p e}\right)$ onset of beam plasma interaction that can seed the SM process. Seeding can occur because this onset corresponds to the driving of initial plasma wakefields starting at the RIF and with amplitudes depending on the local bunch density $[14,15]$.

The train of microbunches resulting from the SM process leaves the plasma after $10 \mathrm{~m}$ and passes through an aluminum-coated screen where protons emit optical transition radiation (OTR), $3.5 \mathrm{~m}$ from the plasma exit. The OTR has the same spatiotemporal structure as the modulated proton bunch. A streak camera resolves the incoming OTR light imaged onto its entrance slit in space and in time with resolutions of $80 \mu \mathrm{m}$ and $\sim 1 \mathrm{ps}$, respectively, over a 73 ps time window. Since the entrance slit is narrower than the bunch radius at the screen location, images display the bunch charge density and not its charge [25]. A transfer line (dashed blue line in Fig. 1 [26]) guides a mirror bleedthrough of the laser pulse to the streak camera. This signal (in red circle in inset 2 of Fig. 1) indicates on each image the relative timing of the RIF within the proton bunch with $0.53 \mathrm{ps}$ (rms) accuracy and $0.16 \mathrm{ps}$ precision. It can be delayed together with the camera trigger signal to appear on the image at times later than that of the RIF, as seen every $50 \mathrm{ps}$ at the bottom of Fig. 4(a). This signal is necessary to refer images in time with respect to the RIFs and with respect to each other's timing, because the streak camera triggering system has a time jitter of $4.8 \mathrm{ps}$ (rms), equivalent to approximately half a period of the wakefields.

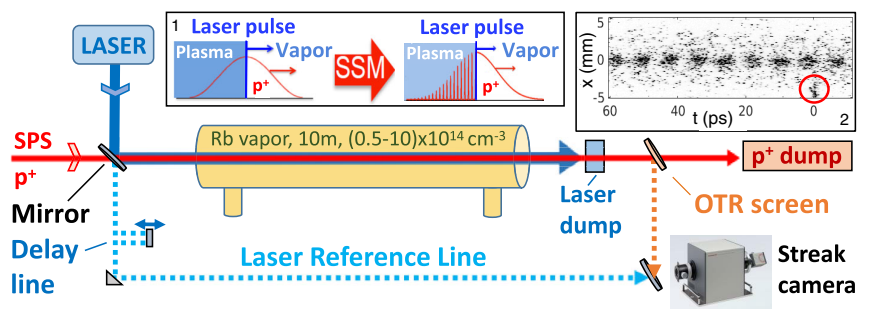

FIG. 1. Schematic of the experimental setup showing the main components used for measurements presented here. Inset 1: RIF in the middle of the proton bunch $\left(t_{\mathrm{RIF}}=0 \mathrm{ps}\right)$. Inset 2: streak camera image of a modulated proton bunch, laser reference signal at $t=0 \mathrm{ps}$ (red circle).
In the following, we refer to this signal as the laser reference signal (LRS).

Results.-We observe that when we use the RIF for plasma creation only, placing it nano- to microseconds ahead of the proton bunch, SM occurs [27]. In this case SM can grow from noise present in the system. The wakefield amplitude driven by shot noise in the proton bunch distribution was estimated at the tens of $\mathrm{kV} / \mathrm{m}$ level [28]. The laser pulse drives wakefields at the $<100 \mathrm{kV} / \mathrm{m}$ level at the plasma densities of these experiments [29]. Figure 2(a) shows a composite image of the time structure of the center part of the modulated proton bunch (compare Fig. 1, inset 2) for ten events in the 73 ps window, placed $150 \mathrm{ps}\left(0.6 \sigma_{t}\right)$ ahead of the bunch peak. These events are aligned in time with respect to the LRS. The LRS alignment procedure yields a $\sim 50$-ps-long common window between images. The LRS (not shown) is placed at $t=0 \mathrm{ps}$ on each image. The RIF is 600 ps $\left(2.4 \sigma_{t}\right)$ ahead of the bunch peak (i.e., $450 \mathrm{ps}, 1.8 \sigma_{t}$ between RIF and $t=0$ on the image). Each image is normalized to its incoming bunch population. The figure clearly shows that from event to event microbunches appear at no particular times with respect to the RIF. It also shows that the measured microbunch charge density varies considerably. Variations in bunch density on these images can be attributed to amplitude variations of focusing and defocusing fields [25]. Variations in timing or phase and amplitude of the modulation are expected for the occurrence of a (nonseeded) instability such as SMI [11].

Figure 2(b) shows a similar plot to that of Fig. 2(a), but with the RIF placed closer, $350 \mathrm{ps}\left(1.4 \sigma_{t}\right)$ ahead of the bunch peak and thus with larger wakefield amplitude at the RIF, with all other parameters unchanged. It is clear that in this case the microbunches appear essentially at the same time with respect to the RIF and with much more consistent charge density than in the previous case. These data show the behavior expected from a seeded process such as SSM. From these two plots we conclude that in the first case the
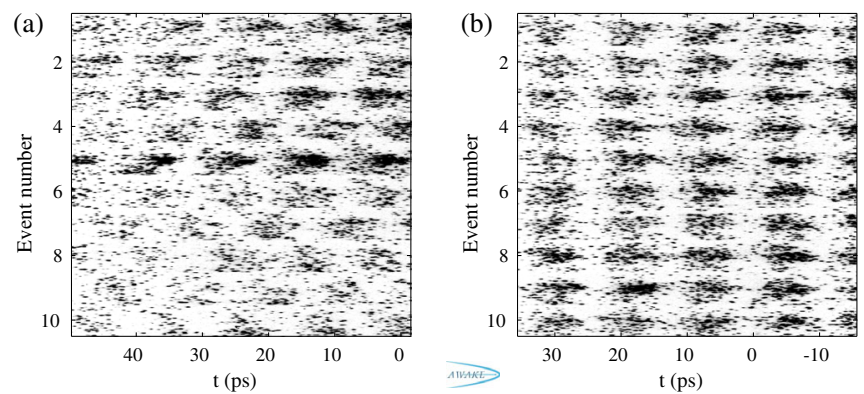

FIG. 2. Composite images of the center part of the streak camera image (see Fig. 1, inset 2) for ten events with (a) RIF $600 \mathrm{ps}\left(2.4 \sigma_{t}\right)$ and (b) RIF $350 \mathrm{ps}\left(1.4 \sigma_{t}\right)$ ahead of the proton bunch center. Front of the bunch on the right-hand side. Events aligned with respect to LRS ([26], at $t=0$, not visible). Both cases: LRS $150 \mathrm{ps}\left(0.6 \sigma_{t}\right)$ ahead of bunch center, $n_{e 0}=0.94 \times 10^{14} \mathrm{~cm}^{-3}$. 
phase of the modulation is not reproducible from event to event (SMI), whereas it is in the second case (SSM).

In order to quantify the observed effect, we determine the phase or timing (using the modulation frequency or period) of the bunch modulation with respect to the RIF. For this purpose we sum counts of the bunch image in a $\cong \pm 430-\mu \mathrm{m}$-wide region around the axis of the bunch at the OTR screen to obtain a time profile of the bunch SM. At this location the incoming bunch transverse rms size is $\cong 574 \mu \mathrm{m}$ [see Fig. 4(a), $t<0 \mathrm{ps}$ ]. For each event, we determine the time of the LRS in the 73 ps window. We calculate the relative phase or timing of the microbunch appearing after the LRS as explained in the Supplemental Material [30]. For the dataset analyzed here $\left(n_{e 0}=\right.$ $0.94 \times 10^{14} \mathrm{~cm}^{-3}$ ), the modulation frequency is $87.1 \mathrm{GHz}$.

Figure 3 shows the variation in relative phase for six series (including the events of Fig. 2) of approximately 18 events each, measured with the analysis window (and LRS) $150 \mathrm{ps}$ ahead of the bunch peak, as a function of the RIF timing $t_{\mathrm{RIF}}$ along the bunch normalized to the rms bunch duration. The phase distributions for $t_{\mathrm{RIF}} \geq 2.0 \sigma_{t}$ cover a range (blue diamonds) close to $2 \pi$ and their rms (blue circles) approaches the value expected for a uniform distribution, 29\%. This corresponds to a phase randomly distributed from event to event, possibly varying over more than $2 \pi$. On the contrary, for $t_{\mathrm{RIF}} \leq 1.8 \sigma_{t}$, the ranges are $\ll 2 \pi$ and their rms is small, $\sim 6 \%$, which shows that the phase of the SM is reproducible from event to event (within the rms range). This is the transition from SMI, with the modulation phase not reproducible [Fig. 2(a)], to SSM, with the modulation phase reproducible within a small range of $2 \pi$ [Fig. 2(b)], when the initial wakefield amplitude increases. We show later, by delaying the observation window timing for a fixed $t_{\mathrm{RIF}}$, that when reached in one

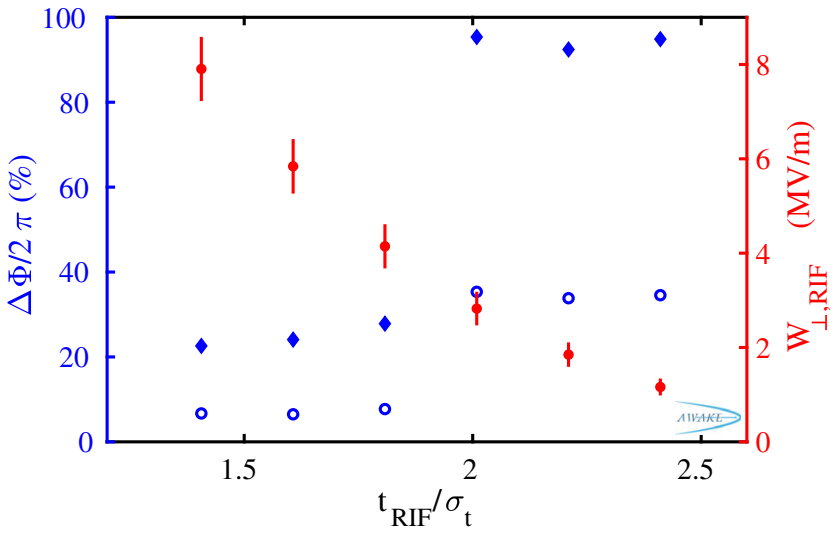

FIG. 3. Measured rms (blue circles) and full phase variation (blue diamond), and initial linear transverse wakefield amplitude (filled red circles) as a function of $t_{\mathrm{RIF}}$ normalized to $\sigma_{t}$. The error bars indicate the statistical uncertainty of $10.1 \%$ (see text). Error bars representing the uncertainty in $t_{\mathrm{RIF}}$ due to the $15 \mathrm{ps}\left(0.06 \sigma_{t}\right)$ rms proton timing jitter are not plotted. Same LRS timing and $n_{e 0}$ as in Fig. 2. window, the timing or phase reproducibility occurs all along the bunch, as expected. In the SMI regime, timeresolved images (not presented here) of the SM near the seed point show that full SM starts at different times along the bunch, unlike in the seeded cases, where it starts at the RIF [14]. This explains the $\sim 2 \pi$ (modulo) phase variations observed with SMI. In the SSM regime, the observed phase rms variations of $\sim 6 \%$ (of $2 \pi$ ) results from at least three main contributions. First, the intrinsic phase variations that are the goal of the measurement. Second, variations of initial parameters from event to event originating from the bunch or the plasma. We measure rms variations in bunch length, $\approx 1.6 \%$, population, $\approx 5 \%$, and plasma density, $<0.2 \%$. There may be additional variations in bunch waist size and location and emittance that we do not monitor for each event. The influence of these variations on the phase can in principle be obtained from numerical simulations [21], though reaching percent level precision is very challenging. Third, variations due to the measurement accuracy influenced by the streak camera resolution of the modulation, the limited number of microbunches per image, signal noise, and uncertainties in determining the position of the LRS ( $0.16 \mathrm{ps})$. As a consequence, the measured variations can only be seen as an upper limit for the real phase variations. They are probably dominated by the last two contributions mentioned, mainly by uncertainties originating from the noisy measured modulation profile (see Supplemental Material [30]).

The initial transverse wakefield amplitude (at the plasma entrance) can be calculated as a function of the RIF timings of Fig. 3: $W_{\perp, \mathrm{RIF}}\left(t=t_{\mathrm{RIF}}\right.$ ) (see Supplemental Material [30]). The initial proton bunch density $\left[n_{b}(t)=n_{b 0} e^{-t^{2} / 2 \sigma_{t}^{2}}\right.$, with $n_{b 0}=1.1 \times 10^{13} \mathrm{~cm}^{-3}$ ] is smaller than the plasma density $\left(n_{e 0}=0.94 \times 10^{14} \mathrm{~cm}^{-3}\right)$. We thus use twodimensional linear plasma wakefield theory [31] to evaluate this amplitude. The modulation period ( $\cong 11.5 \mathrm{ps})$ is much shorter than the rms bunch duration $\left(\sigma_{t}=250 \mathrm{ps}\right)$. We therefore consider the Gaussian bunch density $n_{b}\left(t=t_{\mathrm{RIF}}\right)$ constant over one period behind the RIF and thus $W_{\perp, \mathrm{RIF}}=2\left(m_{e} c^{2} / e\right)\left[n_{b}\left(t_{\mathrm{RIF}}\right) / n_{e 0}\right] d R /\left.d r\right|_{r=\sigma_{r 0}}$. The radial dependence of wakefields through the $R(r)$ coefficient [31] is a function of the transverse bunch profile, considered as Gaussian, and is evaluated at $r=\sigma_{r 0}$, independent of $t$.

We plot the amplitude of $W_{\perp, \mathrm{RIF}}$ for each data point in Fig. 3 (filled red circles). The input parameter variations mentioned above cause a maximum statistical uncertainty of $10.1 \%$ on the field calculation, which includes a $15 \mathrm{ps}$ $\left(0.06 \sigma_{t}\right)$ rms timing jitter between the proton bunch and the laser pulses (RIF and LRS), all added in quadrature. This uncertainty is indicated by the error bars. The plot shows that for the parameters of these experiments, the transition between SMI and SSM occurs between $(2.8 \pm 0.3)$ and $(4.1 \pm 0.4) \mathrm{MV} / \mathrm{m}$. The fact that initial wakefield amplitudes of $(2.8 \pm 0.3) \mathrm{MV} / \mathrm{m}$ do not seed the SM process may indicate that the bunch has density irregularities 


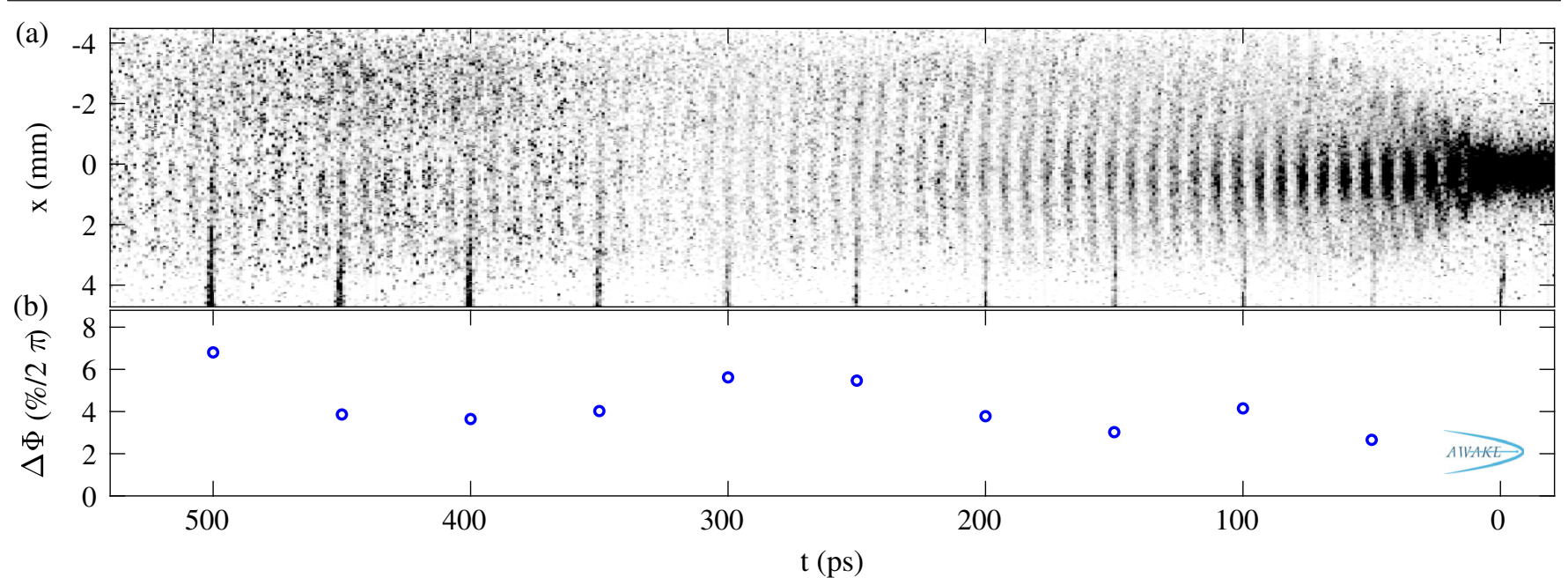

FIG. 4. (a) Time-resolved, "stitched" image of the self-modulated proton bunch with $t_{\mathrm{RIF}}=125 \mathrm{ps}\left(0.5 \sigma_{t}\right), n_{e 0}=1.81 \times 10^{14} \mathrm{~cm}^{-3}$. The RIF is at $t=0$ on the image (not visible). The LRS is visible every $50 \mathrm{ps}$ at the bottom of the image. (b) Modulation rms phase variation for each set of images with equal LRS timing.

driving initial wakefields with amplitude [between $(2.8 \pm 0.3)$ and $(4.1 \pm 0.4) \mathrm{MV} / \mathrm{m}]$ much larger than those of the shot noise assumed in [28] driving $<100 \mathrm{kV} / \mathrm{m}$ fields. These irregularities correspond to $14 \%-20 \%$ of the bunch peak density maintained over at least one period of the wakefields. Since the amplitude of the initial wakefields at the RIF and that of wakefields driven by incoming bunch irregularities follow essentially the same scaling $\left[W_{\perp}(t) \propto n_{b}(t) / n_{e 0}\right]$, we expect the transition from SSM to SMI to occur at the same time along the bunch, independently of the bunch and plasma densities. We also note here that we interpret the reproducibility of the bunch modulation as also that of the wakefields driven toward the end of the plasma, after saturation of the SM process [16]. The wakefield structure is intrinsically linked to the distribution of the self-modulated proton bunch.

The phase reproducibility can be further confirmed by similar phase variation measurements at various delays behind the RIF. Sets of approximately ten images with delay increments of $50 \mathrm{ps}$ between each set were acquired at a higher plasma density $n_{e 0}=1.81 \times 10^{14} \mathrm{~cm}^{-3}$ and a fixed RIF timing of $125 \mathrm{ps}\left(0.5 \sigma_{t}\right)$. Since for these measurements, the RIF is placed much closer to the bunch center $\left(0.5 \sigma_{t}\right)$ than the SSM-SMI transition point determined from the lower plasma density measurements $\left(\sim 1.9 \sigma_{t}\right)$, we expect the SM process to be in the SSM regime. This is confirmed by Fig. 4. Because of the time overlap between sets, all images can be "stitched" together using the LRS as described in Ref. [26] [see Fig. 4(a)]. It is immediately clear from the figure that microbunches of all events align themselves in time or phase and form a coherent modulation of the bunch density over $\sim 2 \sigma_{t}$ behind the RIF. This is only possible when proper seeding is provided (SSM) for each event, relative phase variations between events are small [i.e., all sequences look similar to that of Fig. 2(b)], and the modulation phase is reproducible all along the bunch. All features visible in Fig. 4(a) would wash out if phases were randomly distributed as in Fig. 2(a).

Figure 4(b) shows the result of the phase analysis applied to these events. Over the $\sim 2 \sigma_{t}$ measurement range, larger than the delay from the RIF of $\sim 1 \sigma_{t}$ typically foreseen for external electron injection, the phase variations remain small and in a similar range to those obtained at lower plasma density. Variations along the bunch are most likely due to changes in signal that can be seen in Fig. 4(a) and on individual images, which affects the accuracy of the phase determination. The measured variations remain approximately constant and between 3\% and 7\% (of $2 \pi$ ) all along the bunch.

Summary.-We presented the results of experimental studies of the SM phase for different timings of the RIF with respect to the proton bunch, measured after the 10-m-long plasma. These results demonstrate that the SM process can be seeded; i.e., the phase of the modulation can be defined by the RIF and reproducible from event to event. We observe the transition from phase nonreproducibility and instability (SMI) to seeding and phase reproducibility (SSM) when the transverse wakefield at the RIF exceeds a threshold amplitude, between $(2.8 \pm 0.3)$ and $(4.1 \pm 0.4) \mathrm{MV} / \mathrm{m}$ for $n_{e 0}=0.94 \times 10^{14} \mathrm{~cm}^{-3}$. This value is much larger than that calculated from the bunch shot noise assumed in [28] driving $<100 \mathrm{kV} / \mathrm{m}$ fields. We show that in the SSM regime variations of the modulation phase along the bunch $\left(\sim 2 \sigma_{t}\right)$ are small, measured at $\leq 7 \%$. We attribute most of these small variations to the measurement accuracy of the modulation phase within single, 73 ps time windows including only 6-9 modulation periods. The phase reproducibility also observed at higher plasma density allows for detailed observation of 
the SM process along the whole bunch with $\sim$ ps time resolution [Fig. 4(a)].

Based on these results, one can thus expect that for the studies of electron acceleration during AWAKE Run II [29], the wakefields driven by the bunch train in the second plasma will have a timing or phase also reproducible from event to event since they will be driven by the bunch emerging from the first plasma. Phase reproducibility is required for deterministic acceleration of electrons externally injected into the wakefields, with a fixed delay with respect to the seed.

This work was supported by the Wolfgang Gentner Programme of the German Federal Ministry of Education and Research (Grant No. 05E15CHA); in parts by a Leverhulme Trust Research Project Grant No. RPG2017-143 and by STFC (AWAKE-UK, Cockcroft Institute core and UCL consolidated grants), UK; a Deutsche Forschungsgemeinschaft Project Grant No. PU 213-6/1 "Three-dimensional quasi-static simulations of beam self-modulation for plasma wakefield acceleration"; the National Research Foundation of Korea (No. NRF-2016R1A5A1013277 and No. NRF2020R1A2C1010835); the Portuguese FCT-Foundation for Science and Technology, through Grants No. CERN/ FIS-TEC/0032/2017, No. PTDC-FIS-PLA-2940-2014, No. UID/FIS/50010/2013, and No. SFRH/IF/01635/ 2015; NSERC and CNRC for TRIUMF's contribution; the U.S. National Science Foundation under Grant No. PHY-1903316; and the Research Council of Norway. M. Wing acknowledges the support of DESY, Hamburg. Support of the Wigner Datacenter Cloud facility through the "Awakelaser" project is acknowledged. The work of V. H. has been supported by the European Union's Framework Programme for Research and Innovation Horizon 2020 (2014-2020) under the Marie Sklodowska-Curie Grant Agreement No. 765710. The AWAKE Collaboration acknowledge the SPS team for their excellent proton delivery.

[1] T. Tajima and J. M. Dawson, Phys. Rev. Lett. 43, 267 (1979).

[2] P. Chen, J. M. Dawson, R. W. Huff, and T. Katsouleas, Phys. Rev. Lett. 54, 693 (1985).

[3] P. Chen, J. J. Su, T. Katsouleas, S. Wilks, and J. M. Dawson, IEEE Trans. Plasma Sci. 15, 218 (1987); P. Chen, S. Rajagopalan, and J. Rosenzweig, Phys. Rev. D 40, 923 (1989).

[4] I. Blumenfeld et al., Nature (London) 445, 741 (2007).

[5] M. Litos et al., Nature (London) 515, 92 (2014).
[6] A. J. Gonsalves et al., Phys. Rev. Lett. 122, 084801 (2019).

[7] J. M. Dawson, Phys. Rev. 113, 383 (1959).

[8] Constants have usual meaning: $e$ electron charge, $\varepsilon_{0}$ vacuum permittivity, $m_{e}$ electron mass, $c$ speed of light in vacuum.

[9] J. J. Su, T. Katsouleas, J. M. Dawson, P. Chen, M. Jones, and R. Keinigs, IEEE Trans. Plasma Sci. 15, 192 (1987).

[10] C. J. McKinstrie, Phys. Fluids B 4, 2626 (1992).

[11] N. Kumar, A. Pukhov, and K. Lotov, Phys. Rev. Lett. 104, 255003 (2010).

[12] E. Gschwendtner et al., Nucl. Instrum. Methods Phys. Res., Sect. A 829, 76 (2016).

[13] P. Muggli et al. (AWAKE Collaboration), Plasma Phys. Controlled Fusion 60, 014046 (2018).

[14] AWAKE Collaboration, Phys. Rev. Lett. 122, 054802 (2019).

[15] M. Turner et al. (AWAKE Collaboration), Phys. Rev. Lett. 122, 054801 (2019).

[16] M. Turner et al. (AWAKE Collaboration), Phys. Rev. Accel. Beams 23, 081302 (2020).

[17] AWAKE Collaboration, Nature (London) 561, 363 (2018); AWAKE Collaboration, Phil. Trans. R. Soc. A 377, 20180418 (2019).

[18] Veronica K. Berglyd Olsen, E. Adli, and P. Muggli, Phys. Rev. Accel. Beams 21, 011301 (2018).

[19] S. P. Le Blanc, M. C. Downer, R. Wagner, S.-Y. Chen, A. Maksimchuk, G. Mourou, and D. Umstadter, Phys. Rev. Lett. 77, 5381 (1996).

[20] Y. Fang, V. E. Yakimenko, M. Babzien, M. Fedurin, K. P. Kusche, R. Malone, J. Vieira, W. B. Mori, and P. Muggli, Phys. Rev. Lett. 112, 045001 (2014).

[21] M. Moreira, J. Vieira, and P. Muggli, Phys. Rev. Accel. Beams 22, 031301 (2019).

[22] G. Plyushchev, R. Kersevan, A. Petrenko, and P. Muggli, J. Phys. D 51, 025203 (2018).

[23] E. Öz, and P. Muggli, Nucl. Instrum. Methods Phys. Res., Sect. A 740, 197 (2014).

[24] F. Batsch, M. Martyanov, E. Oez, J. Moody, E. Gschwendtner, A. Caldwell, and P. Muggli, Nucl. Instrum. Methods Phys. Res., Sect. A 909, 359 (2018).

[25] A.-M. Bachmann and P. Muggli, J. Phys. Conf. Ser. 1596, 012005 (2020).

[26] F. Batsch, J. Phys. Conf. Ser. 1596, 012006 (2020).

[27] S. Gessner et al. (AWAKE Collaboration), arXiv:2006 09991.

[28] K. V. Lotov, G. Z. Lotova, V. I. Lotov, A. Upadhyay, T. Tückmantel, A. Pukhov, and A. Caldwell, Phys. Rev. ST Accel. Beams 16, 041301 (2013).

[29] P. Muggli et al., J. Phys. Conf. Ser. 1596, 012008 (2020).

[30] See Supplemental Material at http://link.aps.org/ supplemental/10.1103/PhysRevLett.126.164802 for a description of the analysis that yields the timing or phase of the microbunch train and for the calculation of the initial transverse wakefield amplitude.

[31] R. Keinigs and M. E. Jones, Phys. Fluids 30, 252 (1987). 